\title{
The Electrostatic Interaction of a Charged Particle with a Surface: The Effect of Surface Charge Rearrangement
}

\author{
Heng-Kwong Tsao*,1 and Yu-Jane Sheng $\dagger$ \\ * Department of Chemical Engineering, National Central University, Chung-li, Taiwan 320, Republic of China; and $\dagger$ Department \\ of Chemical Engineering, National Taiwan University, Taipei, Taiwan 106, Republic of China
}

Received June 5, 2000; accepted September 28, 2000

\begin{abstract}
In this paper we investigate the electric interaction between a charged particle and a surface in which the charged ions are capable of moving in responseto theelectric potential disturbancecaused by the approach of the charged particle. Such surfaces include ionic surfactants distributed in air-water interface and charged lipids in bilayer membranes. On the basis of the mean field theory, the free energy of the system, which includes the electrostatic internal energy and the entropy of the mobile ions and surface ions, can be written down. The surface charge-potential relation is then derived by the calculus of variation. When the potential disturbance is small enough, a linear charge regulation model is obtained. The interaction energy associated with a long rod parallel to the interface is studied and an analytical expression is obtained. When a rod approaches an oppositely charged surface, the interaction can change from attraction to repulsion, depending on the ratio of the characteristic regulation length to the $D$ ebye length. At low surface charge density, the surface behaves as under the condition of constant charge density and acts as that of constant potential for high enough charge density.
\end{abstract}

Key Words: free energy; Poisson-Boltzman equation; surface ions rearrangement; charge regulation.

\section{INTRODUCTION}

The electrostatic interactions between a charged particle and a charged surface have been an important subject of both experimental and theoretical investigations due to their practical applications in a variety of fields, such as protein adsorption and the adhesion of DNA to substrates. Kaplan et al. have investigated the interaction between a charged sphere and a charged planar wall under condition of constant surface potential $(1,2)$. When both surfaces are maintained at constant surface charge density, Stahlberg et al. calculated the electrostatic interaction energy by solving the linearized Poisson-Boltzmann equation (3). For an ion-penetrable sphere interacting with an ion-impenetrable plate, an exact expression of the electrostatic interaction energy has been given by Ohshima and Kondo for both the constant surface potential and the constant surface charge density cases (4).

\footnotetext{
${ }^{1}$ To whom correspondence should be addressed.
}

Nonspherical colloidal particles are also plentiful in nature and industrial applications. A large class of macromolecules, which are not flexible, is assumed to have a rod-like shape. For instance, some polynucleotides or polypeptides form a helix structure that can be considered as a rigid rod. Recently, applications involving DNA strands and charged surfaces, such as prokaryotic DNA replication, to gene therapy, and even DNA chip technology, have raised an interest in understanding the interactions between charged rods and charged surfaces. In contrast to spherical particles, little attention has been focused on the electrostatic interactions of nonspherical particles with a charged surface (5-7).

When a charged particle approaches a charged surface, the plane may maintain constant surface potential or constant surface charge density during interactions (8). In between these two limits, the surface charge density can be regulated by the dissociation of the counterions, i.e., the potential determining ions. The electric field distrubance due to the approach of the charged particle influences the local degree of dissociation on the interface and therefore alters the surface charge distribution. Although the charges on a solid plane often originate from dissociation of counterions or adsorption of ions, some charged surfaces, such as air-water interfaces and biomembranes, contain the surface ions that are mobile in the two-dimensional plane. For such surfaces, consequently, the approach of a charged particle can also induce the redistribution of charges at the interface. The total charges at the surface, however, are still maintained the same.

The calculation of the double-layer interaction between a charged particle and a charged surface is a problem of considerable intricacy. Though theoretical progress has been made by the statistical mechanical theories, such as Brownian dynamics simulations $(6,7)$, the mean-field descriptions, such as the Poisson-Boltzmann equation (5), are also useful tools because of their concise forms. For a surface capable of charge regulation, the latter approach is particularly advantageous. In conventional charge-regulation models, such as the two-site dissociation model, the charge-potential relations can be obtained directly from the law of mass action (9-12). However, for a surface in which the charges can rearrange themselves, the surface charge-potential relation is not known and must be determined by minimization of the free energy of the system. 
In this paper, we consider a surface in which the charged ions are capable of moving in response to the electric potential disturbance due to the approach of a charged particle. On the basis of the mean-field description, we write down the free energy of the system, which contains the electrostatic internal energy, the bulk entropy of the mobile ions, and the two-dimensional entropy of the surface ions. The Poisson-Boltzmann equation and the surface charge-potential relation are then derived through minimization of the free energy by the calculus of variation. The electrostatic interactions of a long rod with a surface are then considered under the Debye-Hückel approximation.

\section{FREE ENERGY AND CHARGE REGULATION}

A system consists of charged ion-impenetrable surfaces $(S)$ and charged particles immersed in $z_{+}: z_{-}$electrolyte solutions $(V)$. The charges on the particles are regarded as fixed charges. When the charged particle approaches the surface, the surface ions can move and rearrange themselves in response to the electric potential disturbance.

The free energy of the system $F$ contains the electrostatic internal energy contribution $U_{\mathrm{el}}$ and the entropic contribution $-T S_{\mathrm{el}}$,

$$
F=U_{\mathrm{el}}-T S_{\mathrm{el}}
$$

Within the mean-field approximation, the total free energy can be expressed in terms of the local electric potential $\psi(\mathbf{r})$, the concentrations of the mobile ions $c_{ \pm}(\mathbf{r})$, the distribution of fixed charges $\rho_{\mathrm{f}}(\mathbf{r})$, and the surface charge density $\sigma(\mathbf{r})$. Assume that the electrolyte is a dilute, ideal solution with uniform dielectric properties. In addition, the surface ions also behave ideally. As a result,

$$
\begin{aligned}
U_{\mathrm{el}}= & -\frac{\varepsilon_{\mathrm{r}} \varepsilon_{0}}{2} \int_{V}|\nabla \psi|^{2} d \mathbf{r}^{3}+\int_{V}\left[z_{+} e c_{+}(\mathbf{r})+z_{-} e c_{-}(\mathbf{r})\right. \\
& \left.+\rho_{\mathrm{f}}(\mathbf{r})\right] \psi(\mathbf{r}) d \mathbf{r}^{3}+\int_{S} \sigma(\mathbf{r}) \psi(\mathbf{r}) d \mathbf{r}^{2},
\end{aligned}
$$

and

$$
\begin{aligned}
-T S_{\mathrm{el}}= & k_{\mathrm{B}} T \int_{V}\left\{c_{+}\left[\ln \left(c_{+} v_{0}\right)-1\right]+c_{-}\left[\ln \left(c_{-} v_{0}\right)-1\right]\right\} d \mathbf{r}^{3} \\
& +k_{\mathrm{B}} T \int_{S} \frac{\sigma}{z_{\mathrm{s}} e}\left[\ln \left(\frac{\sigma}{z_{\mathrm{s}} e} a_{0}\right)-1\right] d \mathbf{r}^{2}
\end{aligned}
$$

where $v_{0}$ and $a_{0}$ are the volume per mobile ion and the area per charged molecule composing the surface. $\varepsilon_{\mathrm{r}}$ and $\varepsilon_{0}$ are, respectively, the relative permittivity of the electrolyte solutions and the permittivity of a vacuum. $k_{\mathrm{B}} T$ denotes the thermal energy, $e$ is the electron charge, and $z_{\mathrm{s}}$ is the valency of surface ions. The first term in Eq. [2] is the self-energy of the electic field (13). The next two terms are the electrostatic energy associated with the mobile ions and fixed charges in $V$ and surface ions at $S$. In
Eq. [3] the first term represents the entropies of the mobile ions, whereas the second term is the entropy associated with the ions at the surfaces.

The thermodynamic equilibrium state corresponds to the minimum of the total free energy subject to the constraints of electroneutrality in the system and the conservation of surface ions. That is,

$$
\int_{V}\left(z_{+} c_{+}+z_{-} c_{-}\right) e d \mathbf{r}^{3}+\int_{S} \sigma d \mathbf{r}^{2}=0
$$

and

$$
\int_{S} \sigma(\mathbf{r}) d \mathbf{r}^{2}=\sigma_{0} \int_{S} d \mathbf{r}^{2}
$$

Using the method of Lagrangian multiplier, the variation of the total free energy with respect to $\psi, c_{ \pm}$, and $\sigma$ yields a set of equations which describe the equilibrium state of the system. $\delta F / \delta \psi=0$ gives

$$
\begin{gathered}
\int_{V}\left[\varepsilon_{\mathrm{r}} \varepsilon_{0} \nabla^{2} \psi+z_{+} e c_{+}+z_{-} e c_{-}+\rho_{\mathrm{f}}\right] d \mathbf{r}^{3} \\
+\int_{S}\left[\sigma+\varepsilon_{\mathrm{r}} \varepsilon_{0} \nabla \psi \cdot \mathbf{n}\right] d \mathbf{r}^{2}=0 .
\end{gathered}
$$

The Poisson equation and the boundary condition at $S$ are then obtained,

$$
\begin{gathered}
\nabla^{2} \psi=-\frac{z_{+} e c_{+}(\mathbf{r})+z_{-} e c_{-}(\mathbf{r})+\rho_{\mathrm{f}}(\mathbf{r})}{\varepsilon_{\mathrm{r}} \varepsilon_{0}}, \quad \mathbf{r} \in V, \\
\varepsilon_{\mathrm{r}} \varepsilon_{0} \nabla \psi \cdot \mathbf{n}=-\sigma(\mathbf{r}), \quad \mathbf{r} \in S .
\end{gathered}
$$

$\delta F / \delta c_{ \pm}=0$ produces the Boltzmann distribution,

$$
c_{ \pm}=c_{\mathrm{b}} \exp \left[-\frac{z_{ \pm} e}{k_{\mathrm{B}} T} \psi(\mathbf{r})\right], \quad \mathbf{r} \in V .
$$

The surface charge distribution due to surface ions rearrangement follows the condition $\delta F / \delta \sigma=0$. The Lagrangian multiplier can be evaluated by Eq. [5] and the result is also Boltzmannlike,

$$
\sigma(\mathbf{r})=\frac{\sigma_{0}}{\left\langle\exp \left[-\frac{z_{\mathrm{s}} e}{k_{\mathrm{B}} T} \psi\right]\right\rangle_{\mathrm{s}}} \exp \left[-\frac{z_{\mathrm{s}} e}{k_{\mathrm{B}} T} \psi(\mathbf{r})\right], \quad \mathbf{r} \in S,
$$

where $c_{\mathrm{b}}$ is the bulk concentration of the mobile ions and $\langle\cdot\rangle$ denotes the area average at $S$,

$$
\langle A\rangle=\frac{\int_{S} A(\mathbf{r}) d \mathbf{r}^{2}}{\int_{S} d \mathbf{r}^{2}} .
$$

In the Debye-Hückel limit, Eqs. [8] and [9] can be linearized. As a result, we are left with the linearized Poisson-Boltzmann 
equation and the linearized surface charge-potential relation,

$$
\nabla^{2} \psi=\kappa^{2} \psi-\frac{\rho_{\mathrm{f}}(\mathbf{r})}{\varepsilon_{\mathrm{r}} \varepsilon_{0}}, \quad \mathbf{r} \in V
$$

and

$$
\sigma(\mathbf{r})=\sigma_{0}^{*}\left[1-\frac{z_{\mathrm{s}} e}{k_{\mathrm{B}} T} \psi(\mathbf{r})\right], \quad \mathbf{r} \in S .
$$

Here $\kappa^{-1}$ is the Debye length and

$$
\sigma_{0}^{*}=\sigma_{0}\left[1+\frac{z_{\mathrm{s}} e}{k_{\mathrm{B}} T}\langle\psi\rangle_{\mathrm{s}}\right] .
$$

After obtaining the electric field, $\sigma_{0}^{*}$ and $\langle\psi\rangle_{\mathrm{s}}$ can be determined in a self-consistent way. If the surface area is large compared to the charged particle, $\langle\psi\rangle_{\mathrm{s}}$ is weakly dependent on the distance between the charged particle and the charged plane. Note that in order to fulfill the condition $\sigma(\mathbf{r}) / \sigma_{0}>0$ everywhere, as illustrated in Eq. [9], $z_{\mathrm{s}} e\langle\psi\rangle_{\mathrm{s}} / k_{\mathrm{B}} T>-1$. Equation [12] indicates that the behavior of the surface is similar to that of a surface capable of linear charge regulation due to counterion dissociation.

Now we return to the free energy calculation. Using the divergence theorem and Eq. [8], $U_{\mathrm{el}}$ in Eq. [2] can be simplified furthermore.

$$
\begin{aligned}
U_{\mathrm{el}}= & \frac{1}{2} \int_{V} \rho_{\mathrm{f}}(R) \psi d \mathbf{r}^{3}+\frac{1}{2} \int_{S} \sigma \psi d \mathbf{r}^{2} \\
& +O\left[k_{\mathrm{B}} T \int_{V} c_{\mathrm{b}}\left(\frac{z_{ \pm} e \psi}{k_{\mathrm{B}} T}\right)^{2} d \mathbf{r}^{3}\right] .
\end{aligned}
$$

Similarly, substituting Eqs. [8] and [9] into Eq. [3] gives

$$
\begin{aligned}
-T S_{\mathrm{el}}= & -\int_{S} \sigma \psi d \mathbf{r}^{2}+k_{\mathrm{B}} T \int_{S} \frac{\sigma}{z_{\mathrm{s}} e}\left[\ln \left(\frac{\sigma_{0}^{*} a_{0}}{z_{\mathrm{s}} e}\right)-1\right] d \mathbf{r}^{2} \\
& +O\left[k_{\mathrm{B}} T \int_{V} c_{\mathrm{b}}\left(\frac{z_{ \pm} e \psi}{k_{\mathrm{B}} T}\right)^{2} d \mathbf{r}^{3}\right]
\end{aligned}
$$

For a given distribution of fixed charges, such as rod- and ringlike particles, the variation of the free energy with the separation from the center of fixed charges to charged surfaces, $R$, is independent of the self-energy. Note that $\sigma_{0}^{*}$ is separation-dependent. Using Eqs. [5] and [12] for $\sigma$, the free energy can be expressed as

$$
\begin{aligned}
F(R)= & \frac{1}{2} \int_{V} \rho_{\mathrm{f}}(R) \psi d \mathbf{r}^{3}-\frac{1}{2} \sigma_{0}^{*} \int_{S} \psi d \mathbf{r}^{2}+\int_{S} \sigma_{0}\langle\psi\rangle_{\mathrm{s}} d \mathbf{r}^{2} \\
& +O\left[\left(\frac{e \psi}{k_{\mathrm{B}} T}\right)^{2}\right]+\text { constant }
\end{aligned}
$$

where the constant denotes the separation-independent terms. This result differs from that of a charged surface, which can be linearly regulated by counterion dissociation (9-12). Equation [14b] indicates that the leading order contribution to the entropy comes from that associated with surface ions rearrangement under the condition of $e \psi / k_{\mathrm{B}} T \ll 1$.

\section{A CHARGED ROD PARALLEL TO THE INTERFACE}

Now we apply the above results to the interaction between a rod-like particle and the charged interface. The length of the rod $L$ is large and the diameter $d$ is small compared to the Debye length $\kappa^{-1}$, i.e., $d \ll \kappa^{-1} \ll L$. Consequently, the charged rod can be treated as a line of point charges. It is positioned along the plane, i.e., perpendicular to the $z$-axis as shown in Fig. 1. The line charge density is $\alpha$ and the electric potential in the system is low enough so that the Debye-Hückel approximation is valid.

The surface potential-charge relation in Eq. [12] is the same as that associated with a linear charge-regulation surface due to counterion dissociation $(10,11)$. Thus the distribution of the electric potential can be solved by the same method (14). Because the superposition principle can be used in the DebyeHückel limit, this problem can be reduced to that associated with a fixed point ion carrying charge $q$ at position $\mathbf{Q}$, i.e., $\rho_{\mathrm{f}}(\mathbf{r})=q \delta(\mathbf{Q})$. The electric potential at $\mathbf{P}$ can be written as the sum of three contributions

$$
\psi(\mathbf{P})=\psi_{\mathrm{p}}+\psi_{q}+\psi_{q^{*}}
$$

where $\psi_{\mathrm{p}}$ and $\psi_{q}$ are, respectively, the unperturbed potentials caused by the surface and the fixed charge in the absence of interactions. The last term $\psi_{q^{*}}$ can be considered as the potential produced by the image point with charge $q^{*}$, which must satisfy the charge-potential relation on the surface Eq. [12]. The solutions are given by

$$
\begin{aligned}
& \psi_{\mathrm{p}}=\frac{k_{\mathrm{B}} T / z_{\mathrm{s}} e}{1+\kappa \lambda} \exp (-\kappa z), \\
& \psi_{q}=\frac{q}{4 \pi \varepsilon_{\mathrm{r}} \varepsilon_{0}} \frac{\exp (-\kappa \tilde{s})}{\tilde{s}},
\end{aligned}
$$

and

$$
\begin{aligned}
\psi_{q^{*}}= & -\frac{q \kappa}{4 \pi \varepsilon_{\mathrm{r}} \varepsilon_{0}} \int_{1}^{\infty}\left(\frac{1-\kappa \lambda \cdot \eta}{1+\kappa \lambda \cdot \eta}\right) J_{0} \\
& \times\left(\sqrt{\eta^{2}-1} \cdot \kappa r\right) \exp [-\eta \kappa(R+z)] d \eta,
\end{aligned}
$$

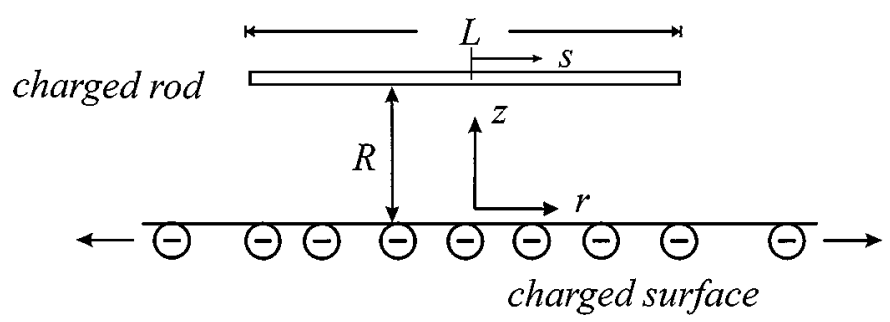

FIG. 1. Schematic representation of the system containing a long rod of length $L$ at a separation $R$ from the charged surface. $s$ is the distance measured from the center of the rod. 
where $\tilde{s}=|\mathbf{P}-\mathbf{Q}|$ and $\lambda=\varepsilon_{\mathrm{r}} \varepsilon_{0} k_{\mathrm{B}} T / \sigma_{0}^{*} z_{\mathrm{s}} e$ represents the characteristic regulation length. The solution of $\psi_{q^{*}}$ is obtained by the method of separation of variables (14). Equations [17]-[19] are the results for a fixed point charge and can be generalized to any string-like particle straightforward.

Now we use the results [17]-[19] to calculate the mean surface potential $\langle\psi\rangle_{\mathrm{s}}$ and to determine $\sigma_{0}^{*}$ and $\lambda$ as a charged rod approaches the surface:

$$
\langle\psi\rangle_{\mathrm{s}}=\frac{k_{\mathrm{B}} T / z_{\mathrm{s}} e}{1+\kappa \lambda}+\frac{\alpha L}{S} \frac{\lambda \exp (-\kappa R)}{\varepsilon_{\mathrm{r}} \varepsilon_{0}(1+\kappa \lambda)} .
$$

Obviously, the second term is small if the surface area $S$ is large enough or the rod is far away from the surface, i.e., $\kappa R \gg 1$. The criterion will be given later. Since the regulation length $\lambda$ is inversely proportional to $\sigma_{0}^{*}$, which in turn depends on $\langle\psi\rangle_{\mathrm{s}}$, it can be decided from Eq. [20] so that

$$
\sigma_{0}^{*}=\sigma_{0}\left(1+\frac{1}{1+\kappa \lambda}\right)
$$

and

$$
\kappa \lambda=\frac{\sqrt{1+4 \Lambda^{2}}+1}{2 \Lambda}-1 .
$$

Here the dimensionless charge density is defined as $\Lambda=$ $\sigma_{0} z_{\mathrm{s}} e / \kappa \varepsilon_{\mathrm{r}} \varepsilon_{0} k_{\mathrm{B}} T$. As $\Lambda \rightarrow \infty, \kappa \lambda \approx 1 / 2 \Lambda \rightarrow 0$. On the other hand, as $\Lambda \rightarrow 0, \kappa \lambda \approx 1 / \Lambda \rightarrow \infty$.

We can also calculate the interaction energy for a rod parallel to a charged surface by substituting Eqs. [17]-[19] into Eq. [15]. The electrostatic interaction energy $W$ is defined as the difference between the free energy at separation $R$ and infinity, i.e., $W(R)=F(R)-F(\infty)$. According to Eq. [15], the interaction energy $W$ can be written as $W=W_{\mathrm{p}}+W_{\mathrm{r}}$. Here $W_{\mathrm{p}}$ is the interaction energy associated with the surface, in which the surface ions can rearrange themselves, and it comes from the potential produced by the charged rod and its image,

$$
\begin{aligned}
W_{\mathrm{p}}= & -\frac{1}{2} \sigma_{0}^{*} \int_{-L / 2}^{L / 2}\left[\int_{0}^{\infty}\left(\psi_{q}+\psi_{q}^{*}\right)_{z=0} 2 \pi r d r\right] \alpha d s \\
& +\int_{S} \sigma_{0}\langle\psi\rangle_{\mathrm{s}} d \mathbf{r}^{2} \\
= & -\frac{1}{2} \frac{\sigma_{0}}{\kappa \varepsilon_{\mathrm{r}} \varepsilon_{0}} \frac{\kappa \lambda(2+\kappa \lambda)}{(1+\kappa \lambda)^{2}}(\alpha L) e^{-\kappa R} \\
& +\frac{\sigma_{0}}{\kappa \varepsilon_{\mathrm{r}} \varepsilon_{0}} \frac{\kappa \lambda}{(1+\kappa \lambda)}(\alpha L) e^{-\kappa R} .
\end{aligned}
$$

$W_{\mathrm{r}}$ is the interaction energy associated with the rod and is due to the potential produced by the plane and the image,

$$
W_{\mathrm{r}}=\frac{1}{2} \int_{-L / 2}^{L / 2} \psi_{\mathrm{p}} \alpha d s+\frac{1}{2} \int_{-L / 2}^{L / 2}\left[\int_{-L / 2}^{L / 2} \psi_{q^{*}} d s\right] \alpha d s^{\prime},
$$

TABLE 1

The Coefficients $b_{k}$ in Eq. [24] for the Interaction E nergy of a Long Rod Parallel to a Charged Surface

\begin{tabular}{cc}
\hline$k$ & $b_{k}(a)$ \\
\hline 1 & $\frac{\sqrt{2}}{2} \frac{1}{1+a}$ \\
2 & $-\frac{\sqrt{2}}{8} \frac{1+5 a}{(1+a)^{2}}$ \\
3 & $\frac{\sqrt{2}}{64} \frac{3+14 a+43 a^{2}}{(1+a)^{3}}$ \\
4 & $-\frac{\sqrt{2}}{256} \frac{5+27 a+1 a^{2}+177 a^{3}}{(1+a)^{4}}$ \\
5 & $\frac{\sqrt{2}}{4096} \frac{35+220 a+642 a^{2}+1276 a^{3}+2867 a^{4}}{(1+a)^{5}}$ \\
\hline
\end{tabular}

where $s$ and $s^{\prime}$ are, respectively, the distances measured from the center of the rod and its image.

The first term in Eq. [23] can be evaluated from Eq. [17] and the result cancels the contribution from the first term in Eq. [22]. To carry out the second integration in Eq. [23] analytically, we assume that the rod is infinitely long, i.e., integrating with respect to $s$ from $-\infty$ to $\infty$. The error due to the end effects would be $O(\kappa L)^{-1}$ small.

$$
\begin{aligned}
& \frac{1}{2} \int_{-L / 2}^{L / 2}\left[\int_{-\infty}^{\infty} \psi_{q^{*}} d s\right] \alpha d s^{\prime}=\frac{1}{2} \frac{(\alpha L)^{2} \kappa}{4 \pi \varepsilon_{\mathrm{r}} \varepsilon_{0}} \\
& \quad \times\left\{K_{0}(2 \kappa R)-2 \sum_{i=1}^{\infty} b_{i}(\kappa \lambda) \frac{\exp (-2 \kappa R)}{(2 \kappa R)^{i-\frac{1}{2}}} \Gamma\left(i-\frac{1}{2}\right)\right\} .
\end{aligned}
$$

The integration is performed in the appendix and the coefficients $b_{\mathrm{i}}$ are given in Table 1. Equation [24] behaves like $K_{0}(2 \kappa R)$ as $\kappa \lambda \rightarrow \infty$ and $-K_{0}(2 \kappa R)$ as $\kappa \lambda \rightarrow 0$. The infinite series in Eq. [24] converges very slowly and the convergence rate can be greatly improved by adopting the Shank transform.

With Eqs. [22], [23], and [24], the interaction energy is then given by

$$
\begin{aligned}
W^{*}(\kappa R ; \kappa \lambda, \Omega)= & \frac{W(\kappa R)}{\left[\frac{(\alpha L)^{2} \kappa}{4 \pi \varepsilon_{\mathrm{r}} \varepsilon_{0}}\right]}=\Omega \frac{\kappa \lambda}{1+\kappa \lambda} e^{-\kappa R}+\frac{1}{2}\left\{K_{0}(2 \kappa R)\right. \\
& \left.-2 \sum_{i=1}^{\infty} b_{i}(\kappa \lambda) \frac{\exp (-2 \kappa R)}{(2 \kappa R)^{i-\frac{1}{2}}} \Gamma\left(i-\frac{1}{2}\right)\right\},
\end{aligned}
$$

where $\Omega=4 \pi \sigma_{0} / \kappa^{2}(\alpha L)$. The dimensionless interaction energy varies with the dimensionless groups: $\Omega, \kappa \lambda$ (or $\Lambda$ ), and $\kappa R$. Note that Eq. [25] reduces to the result of constant surface charge density $\sigma_{0}$ if $\kappa \lambda \rightarrow \infty$ and to that of constant surface potential $\psi_{0}=\left(\sigma_{0} / \kappa \varepsilon_{\mathrm{r}} \varepsilon_{0}\right)[\kappa \lambda /(1+\kappa \lambda)]$ if $\kappa \lambda \rightarrow 0$.

\section{RESULTS AND DISCUSSION}

The air-water interface and the biomembrane are often involved with the surface ions that are mobile in the twodimensional plane. The effect of surface ions rearrangement due 
to the approach of charged particles on the electrostatic interaction is studied. Under circumstances like strong dissociation, the charges of the particle can be regarded as fixed during interactions. By minimizing the free energy of the system, the surface charge-potential relation is obtained in addition to the PoissonBoltzmann equation. The free energy includes the electrostatic internal energy, the bulk entropy of the mobile ions, and the two-dimensional entropy of the surface charges.

As a charged particle approaches, the local surface charge density may be adjusted by several ways. In the conventional charge regulation models, such as single-site and two-site dissociation models (8), the surface charge density is regulated by the chemical equilibrium taking place on the surface. In these models, the positions of reactive sites are usually fixed at surfaces. For a single-site model, such as $A H \rightarrow A^{-}+H^{+}$, the surface charge $A^{-}$is produced if a counterion $H^{+}$is dissociated from the neutral species $A H$. The equilibrium constant is given by $K=\left[A^{-}\right]\left[H^{+}\right] /[A H]$. The surface charge-potential relation follows

$$
\sigma(\mathbf{r})=\frac{\sigma_{\mathrm{t}}}{1+\left[H^{+}\right]_{\mathrm{b}} \exp \left(\frac{z_{\mathrm{s}} e \psi}{k_{\mathrm{B}} T}\right) / K},
$$

where $\sigma_{\mathrm{t}}$ is the total surface charge associated with complete dissociation and $\left[\mathrm{H}^{+}\right]_{\mathrm{b}}$ is the bulk proton concentration.

In the present study, there is no chemical reaction occurring and the electrostatic interaction is regulated by the local rearrangement of the surface charges. Equation (9) shows that the surface charge density follows a Boltzmann distribution with respect to the surface potential. The differences between conventional charge regulation models and the present model can be seen by comparing Eqs. [9] with [26]. For strong dissociation, i.e., $\left[H^{+}\right] / K \ll 1$, Eq. [26] indicates that the surface charge density is essentially constant $\sigma_{\mathrm{t}}$. However, when $\left[H^{+}\right] / K \gg 1$, Eq. [26] can reduce to a form similar to Eq. [9]. As a consequence, both models should have similar behavior in the weak dissociation limit.

When the electric potential is low enough, all the chargeregulation models can be linearized to give the same form, $\sigma(\mathbf{r})=\sigma_{0}^{*}-C \psi$ with $C \geq 0$. For the case of surface charge rearrangement, $C / \sigma_{0}^{*}=z_{\mathrm{s}} e / k_{\mathrm{B}} T$. Moreover, $\sigma_{0}^{*}$ depends on the separation of the charged particle from the interface $R$ because of the variation of $\langle\psi\rangle_{\mathrm{s}}$ with $R$ as shown in Eq. [13]. Under certain circumstances, $\langle\psi\rangle_{\mathrm{s}}$ can be regarded as a constant and the evaluation of $\sigma_{0}^{*}$ is thus simplified. If the charged particle is a long rod, the criterion of taking $\sigma_{0}^{*}$ to be independent of $R$ is obtained by comparing the two terms in Eq. [20]

$$
S \gg \frac{z_{\mathrm{s}} e(\alpha L)}{\kappa \varepsilon_{\mathrm{r}} \varepsilon_{0} k_{\mathrm{B}} T} \kappa \lambda e^{-\kappa R} .
$$

The result indicates that the assumption is valid if the interfacial area $S$ is large enough. When the particle is far away $\left(e^{-\kappa R} \ll 1\right)$ or the mean charge density is high enough $(\kappa \lambda \ll 1$ or $\Lambda \gg 1)$, this simplification is also reasonable.
DNA or polyelectrolytes, such as poly(diallyldimethylammonium) chloride (PDDA), are often modeled as a rigid, charged rod. Under the Debye-Hückel approximation, the interaction energy for a long rod parallel to the charged interface is calculated and given in Eq. [25] for $\kappa L \gg 1$ and $\kappa d \ll 1$. The interaction energy is found to consist of two terms. The first term is associated with the direct interaction between the charge surface and the rod. It is proportional to the product of the unperturbed surface charge density $\sigma_{0}$ and the total charge carried by the $\operatorname{rod} \alpha L$. However, a correction related to the characteristic regulation length $\kappa \lambda$ must be made. Furthermore, it decays exponentially with the distance $\kappa R$. The second term is associated with the rod and its image. The dielectric behind the surface is polarized by the charged rod. The electric field produced by the induced dipoles in the dielectric is represented by the image of the rod. As a result, it is proportional to the square of the total charge associated with the rod and decays approximately as $\exp (-2 \kappa R) / \sqrt{2 \kappa R}$.

In a $1-1$ electrolyte solution of $1-10 \mathrm{mM}$, the Debye length is about $\kappa^{-1} \approx 3-10 \mathrm{~nm}$. A charged rod with diameter $d \lesssim 1 \mathrm{~nm}$ and length $L \gtrsim 50 \mathrm{~nm}$ satisfies the condition $\kappa d \ll 1$ and $\kappa L \gg 1$ approximately. If the line charge density ranges from $\alpha=0.2$ to $1 e / \mathrm{nm}$, then $\alpha L=10-50 e$ for $L=50 \mathrm{~nm}$. A PDDA polymer may be represented by these parameters (7). As illustrated in Eq. [25], the dimensionless interaction energy is determined by the dimensionless groups: $\Omega, \kappa \lambda$, and $\kappa R$. The interaction energy is scaled by $(\alpha L)^{2} \kappa /\left(4 \pi \varepsilon_{\mathrm{r}} \varepsilon_{0}\right)$, which is about $7 k_{\mathrm{B}} T$ for $\alpha L=10 e$ and $k^{-1}=10 \mathrm{~nm}$ at $298 \mathrm{~K}$. With $\sigma_{0}=0.005 \mathrm{C} / \mathrm{m}^{2}$ and $\kappa^{-1}=3 \mathrm{~nm}$, the characteristic charge ratio $\Omega=4 \pi \sigma_{0} \kappa^{-2} /(\alpha L)$ ranges about from \pm 0.3 to \pm 2 and the dimensionless regulation length $\lambda / \kappa^{-1}$ may be as large as 20 for $\alpha L=50 e$ and $\Omega= \pm 0.2$. The distance $R$ is also scaled by $\kappa^{-1}$. Since the rod is regarded as a line charge in the present analysis, the condition $R \gg d$ must be satisfied to have consistent results. The dimensionless separation $\kappa R=0.5$ for $\kappa^{-1}=3 \mathrm{~nm}$ corresponds to $R=1.5 \mathrm{~nm}$, which is in the margin of validity. Therefore, $\kappa R$ begins from 0.5 instead of 0.0 in Figs. 2 and 3.

Figure 2 shows the variation of the dimensionless interaction energy $W^{*}$ with the dimensionless separation $\kappa R$ for various values of $\kappa \lambda$ at $\Omega=-0.2$. Though $\Omega<0$ denotes that the rod and the surface are oppositely charged, the interaction can vary from attraction to repulsion as $\kappa R$ decreases. When the separation is large enough, the direct-interaction term dominates and thus the interaction is attractive. As $\kappa R \rightarrow 0$, the image-related contribution, i.e., the $2 \kappa R$-term, becomes important and depends on the ratio of the characteristic regulation length to the Debye length, $\kappa \lambda$. When $\kappa \lambda \gg 1$, the surface behaves like under the condition of constant surface charge density. As a result, the repulsion eventually dominates with decreasing the separation. On the contrary, the surface acts like under the condition of constant surface potential for $\kappa \lambda \rightarrow 0$ and the image-related contribution enhances the attraction. The separation at which the interaction force becomes zero is increased with increasing $\kappa \lambda$. In other words, the charge regulation results in an increase of attraction as $\kappa \lambda$ decreases. 


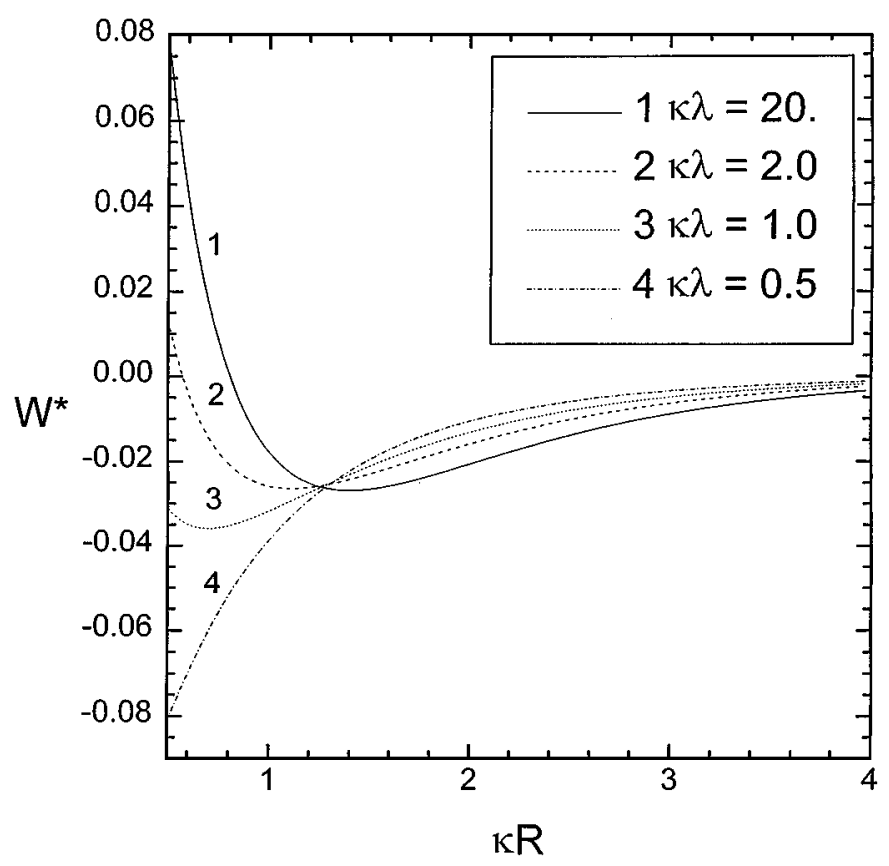

FIG. 2. The variation of the dimensionless interaction energy $W^{*}$ with the dimensionless separation $\kappa R$ for various values of $\kappa \lambda$ at $\Omega=-0.2$.

For a given electrolyte concentration, the regulation length increases with decreasing the average surface charge density according to Eq. [21]. As a consequence, the surface behaves like that of constant charge density for low charge density and functions as that of constant potential if the charge density is

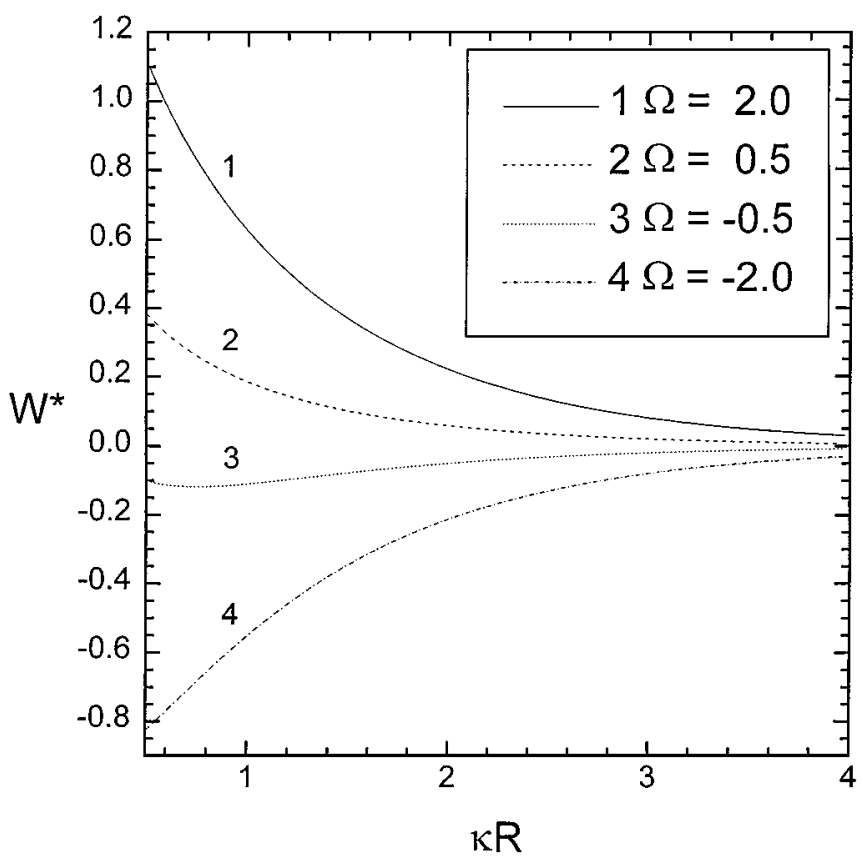

FIG. 3. The variation of the dimensionless interaction energy $W^{*}$ with the dimensionless separation $\kappa R$ for $\Omega= \pm 0.5$ and \pm 2 at $\kappa \lambda=4$. high. For $\kappa \lambda \ll 1$, the Debye-Hückel approximation may be invalid because $\Lambda \rightarrow \infty$ as $\kappa \lambda \rightarrow 0$. The nonlinear form for both the Poisson-Boltzmann equation [6] and the surface chargepotential relation [9] should be adopted. Nevertheless, we expect that the linearized equations can still furnish us with detailed insight and reveal important features of the problem.

Regardless of the sign of $\Omega$, the image-related term always gives the same contributions for a given value of $\kappa \lambda$. As a result, the interaction energy behaves asymmetrically for $\pm \Omega$. Figure 3 depicts the comparison between the interaction energy of $\Omega= \pm 0.5$ and \pm 2 . Since the image-related term is positive for $\kappa \lambda=4$, the rearrangement of surface ions tends to reduce the attraction and to enhance the repulsion between the charged rod and the interface. However, the situation can be reversed if $\kappa \lambda$ is small enough. Note that the image-related term becomes dominant at short separation $\kappa R<1$. When the salt concentration is high enough, the size of the rod can play an equally important role and the interaction may differ from our prediction.

In this study, the surface ions are restricted to move in the twodimensional plane and the total charges are maintained constant during interactions. Nevertheless, the surface ions, such as ionic surfactants, may be in thermodynamic equilibrium with those in the bulk solution. Thus, an electric potential disturbance on the surface can also lead to a redistribution of these ions between the surface and the bulk. In that case, the free energy should include contributions of ionic surfactants in the bulk. A similar approach can be employed to determine the surface charge-potential relation. However, the Lagrangian multiplier must be determined by the conservation of total surfactants. If the charge species is essentially insoluble in water, such as charged lipid, then our analysis is valid.

\section{SYMBOLS}

$\alpha \quad$ line charge density $(\mathrm{C} / \mathrm{m})$

$\delta \quad$ Dirac function

$\varepsilon \quad$ permittivity $\left(\mathrm{C}^{2} / \mathrm{J} \cdot \mathrm{m}\right)$

$\kappa \quad$ Debye-Hückel parameter $\left(\mathrm{m}^{-1}\right)$

$\lambda$ characteristic regulation length (m)

$\rho$ fixed charge density $\left(\mathrm{C} / \mathrm{m}^{3}\right)$

$\sigma \quad$ surface charge density $\left(\mathrm{C} / \mathrm{m}^{2}\right)$

$\psi \quad$ electric potential $(\mathrm{J} / \mathrm{C})$

$\Gamma$ Gamma function

$\Lambda$ dimensionless surface charge density

$\Omega$ characteristic charge ratio of surface to rod

$c$ concentration of mobile ions $\left(\# / \mathrm{m}^{3}\right)$

$d$ diameter of rods (m)

$e$ fundamental charge, $1.6 \times 10^{-19} \mathrm{C}$

$z \quad$ valency of ions

$K$ modified Bessel function

$L$ length of rods (m)

$R$ rod-surface separation (m)

$W \quad$ interaction energy $(\mathrm{J})$ 


\section{APPE NDIX}

We perform the integration of Eq. [24],

$$
\begin{aligned}
& \frac{1}{2} \int_{-L / 2}^{L / 2}\left[\int_{-\infty}^{\infty} \psi_{q^{*}} d r\right] \alpha d r^{\prime} \\
& =-\frac{(\alpha L)^{2} \kappa}{4 \pi \varepsilon_{\mathrm{r}} \varepsilon_{0}} \int_{1}^{\infty}\left(-1+\frac{2}{1+\kappa \lambda \cdot \eta}\right) \frac{\exp (-\eta \cdot 2 \kappa R)}{\sqrt{\eta^{2}-1}} d \eta \\
& =\frac{\alpha^{2} L}{4 \pi \varepsilon_{\mathrm{r}} \varepsilon_{0}}\left[K_{0}(2 \kappa R)-2 \int_{1}^{\infty}\left[\frac{1}{(1+\kappa \lambda \cdot \eta) \sqrt{\eta^{2}-1}}\right]\right. \\
& \left.\quad \times e^{-\eta \cdot 2 \kappa R} d \eta\right]
\end{aligned}
$$

where we have used the relations $\int_{0}^{\infty} J_{0}(b r) d r=b^{-1}$ and $K_{0}$ is the modified Bessel function.

By expanding the preexponential part of the integrand in the last integration in Eq. [A1] about $\eta-1$, one has

$$
\frac{1}{1+\kappa \lambda \cdot \eta} \frac{1}{\sqrt{\eta^{2}-1}}=\sum_{k=1}^{\infty} b_{k}(\kappa \lambda)(\eta-1)^{k-\frac{3}{2}} .
$$

Then the integration can be carried out

$$
\begin{aligned}
& \int_{1}^{\infty} \frac{\exp (-\eta \cdot 2 \kappa R)}{(1+\kappa \lambda \cdot \eta) \sqrt{\eta^{2}-1}} d \eta \\
& \quad=\sum_{k=1}^{\infty} b_{k}(\kappa \lambda) \int_{1}^{\infty}(\eta-1)^{k-\frac{3}{2}} e^{-\eta \cdot 2 \kappa R} d \eta \\
& \quad=\sum_{k=1}^{\infty} b_{k}(\kappa \lambda) \frac{\exp (-2 \kappa R)}{(2 \kappa R)^{k-\frac{1}{2}}} \Gamma\left(k-\frac{1}{2}\right),
\end{aligned}
$$

where we have used the relation

$$
\begin{array}{r}
\int_{1}^{\infty}(\eta-1)^{\frac{v}{2}} e^{-\eta \cdot 2 \kappa R} d \eta=\frac{\exp (-2 \kappa R)}{(2 \kappa R)^{1+\frac{v}{2}}} \Gamma\left(1+\frac{v}{2}\right), \\
v=-1,1,3, \ldots
\end{array}
$$

Some of the coefficients $b_{k}$ are listed in Table 1 . Note that $b_{k}(\alpha) \rightarrow 0$ as $a \rightarrow \infty$ and Eq. [A3] becomes 0 . On the other hand, one has the asymptotic expression of $K_{0}(2 \kappa R)$ from Eq. [A3] as $\kappa \lambda=0$.

\section{ACKNOWLEDGMENT}

This research is supported by National Council of Science of Taiwan under Grant NSC 90-2214-E-008-012.

\section{REFERENCES}

1. Kaplan, F. S., Solomak, T. B., and Brosov, K. N., Kolloid. Zh. 51, 867 (1989).

2. Kaplan, F. S., Solomak, T. B., and Brosov, K. N., Kolloid. Zh. 51, 1100 (1989).

3. Stahlberg, J., Appelgren, U., and Jönsson, B., J. Colloid Interface Sci. 176, 397 (1995).

4. Ohshima, H., and Kondo, T., J. Colloid Interface Sci. 157, 504 (1993).

5. Tsao, H.-K., J. Colloid Interface Sci. 202, 527 (1998).

6. Mashl, R. J., and Gronbech-Jensen, N., J. Chem. Phys. 109, 4617 (1998).

7. Mashl, R. J., Gronbech-Jensen, N., Fitzsimmons, M. R., Lütt, M., and Li, D., J. Chem. Phys. 110, 2219 (1999).

8. Hunter, R. J., "Foundations of Colloid Science.” Oxford, New York, 1992.

9. Healy, T. W., Chan, D., and White, L. R., Pure Appl. Chem. 52, 1207 (1980).

10. Chan, D. Y. C., and Mitchell, D. J., J. Colloid Interface Sci. 95, 193 (1983).

11. Carnie, S. L., and Chan, D. Y. C., J. Colloid Interface Sci. 161, 260 (1993).

12. Tsao, H.-K., and Sheng, Y.-J., Langmuir 14, 6793 (1998).

13. Borukhov, I., and Andelman, D., Phys. Rev. Lett. 79, 435 (1997).

14. Tsao, H.-K., Langmuir 16, 7200 (2000). 